\title{
MATÉRIAUX SUPRACONDUCTEURS ET APPLICATIONS DE LA SUPRACONDUCTIVITÉ
}

\author{
Y. A ROCHER
}

\author{
C. I. T., Alcatel, 91, Bruyères-le-Châtel
}

\begin{abstract}
Résumé. - Les propriétés des matériaux supraconducteurs actuellement disponibles sont examinées en regard des applications dont le développement paraît maintenant presque assuré dans le domaine de la cryoélectrotechnique. Les conducteurs multifilamentaires se prêtent à la construction d'une première génération d'équipements, mais le pas décisif, permettant notamment les applications en régime alternatif sous champ élevé, requiert la mise au point de composites à structure très fine d'un type nouveau.
\end{abstract}

\begin{abstract}
Properties of superconducting materials are reviewed with respect to those applications which appear likely to develop in the near or less near future. Although multifilament conductors have brought considerable improvement and seem to be technically adequate for DC or very low frequency applications, work must be promoted toward new materials that should be characterized by a very fine composite structure.
\end{abstract}

1. Un nouveau départ pour la supraconductivité. L'avenir des applications de la supraconductivité apparaît maintenant plus clair. En dehors des applications déjà traditionnelles, quatre grands domaines ont franchi favorablement le cap des pré-études économiques et techniques et offrent des perspectives d'importants développements industriels : les aimants pulsés, les alternateurs, le transport de courant, les dispositifs de commutation.

Les AIMANTS PULSÉs. - Le projet d'équipement de l'accélérateur super CERN avec des aimants supraconducteurs ne connaîtra de décision définitive qu'en 1974. On peut estimer que, sur une base technique au moins, les chances d'acceptation sont bonnes puisque les nouveaux composites NbTi ont donné des résultats satisfaisants. La mise en place commencerait en 1975.

Les ALTERNATeurs. - Les données économiques sont favorables à la construction d'alternateurs de puissance supérieure à $1000 \mathrm{MW}$. Les besoins et la tendance se prêtent à un démarrage quasi immédiat des études. Dans un premier temps, on pense à un alternateur à inducteur mobile supraconducteur, la solution tout supraconducteur (inducteur + induit) exigeant de sérieux progrès dans le domaine du comportement des matériaux en régime alternatif.

LE TRANSPORT DE COURANT. - Selon les pays et leurs structures de production et de distribution du courant électrique, on envisage la mise en place sur le réseau de câbles de transport supraconducteurs à des dates comprises entre 1985 (G. B.) et 1990-1995
(France). Ces installations seraient destinées à l'entrée dans les grandes agglomérations.

LES DISPOSITIFS DE COMMUTATION. - Le développement de ces dispositifs est en cours pour le stockage d'énergie. En outre, à partir du moment où seront mis en service des matériels de production et de transport du courant électrique utilisant les supraconducteurs, fonctionnant donc à très basse température, il sera très intéressant d'intégrer les dispositifs de transformation, de commande et de contrôle du courant, c'est-à-dire des redresseurs, des commutateurs, des disjoncteurs et des limiteurs de courant.

Pour la première fois, les possibilités d'application de la supraconductivité font apparaître des développements industriels étalés du relativement court terme (aimants CERN) au long terme (câbles). Cet échelonnement profitera à la technique cryogénique et aussi à l'électrotechnique supraconductrice : c'est ainsi, par exemple, que de nombreux problèmes posés par les cryoalternateurs auront été résolus lors de la construction des aimants pulsés du CERN. Cette situation particulièrement favorable devrait encourager les industriels - métallurgistes, câbliers, constructeurs d'aimants, cryogénistes, etc... - et l'Etat à mettre en place l'effort important qui s'impose.

Par ailleurs, il est tenté actuellement d'organiser à l'échelle européenne une concertation de la recherche dans le domaine des supraconducteurs. Un groupe d'experts a préparé un programme préliminaire dans la ligne des perspectives décrites plus haut. Ce programme, prudent et limité, a reçu l'accord des res- 
ponsables politiques et va pouvoir entrer dans une phase active, notamment en ce qui concerne l'évaluation des possibilités de construction d'un prototype de cryoalternateur.

2. Les matériaux. - Les matériaux actuellement disponibles permettent-ils d'envisager raisonnablement les applications qui viennent d'être citées, et notamment celles qui jmpliquent un régime alternatif, pulsé ou transitoire ? On peut constater une évolution prometteuse dans ce sens en reconnaissant la nécessité de progrès très importants dont la voie pourrait être ouverte par des initiatives originales vers des matériaux nouveaux. Il convient de faire le point de ces progrès, de jauger la limite des possibilités offertes par les matériaux "classiques » tels que les $\mathrm{NbTi}$ et définir l'orientation à donner aux études de matériaux pour aller au-delà.

2.1 LeS MATÉRIAUX ClASSiQues. - Il a déjà été souligné que la réalisation des aimants type CERN ne semble pas poser de problèmes majeurs avec les matériaux actuellement disponibles, c'est-à-dire les composites multifilamentaires transposés en $\mathrm{NbTi}$. Ces composites $\mathrm{NbTi}$, voire des rubans en $\mathrm{Nb}_{3} \mathrm{Sn}$ pourraient constituer l'âme supraconductrice des câbles de transport de courant en régime continu, tandis que pour ce même transport en régime alternatif, on envisage l'utilisation de niobium pur.

Les autres applications ne se présentent pas de ce point de vue sous un jour aussi favorable, et notamment les applications aux machines tournantes. Pour envisager un alternateur à induit supraconducteur, il faudrait en effet que les pertes en régime alternatif $(50 \mathrm{~Hz})$ soient réduites d'au moins deux ordres de grandeur par rapport au niveau actuel. Dans un alternateur où seul l'inducteur serait supraconducteur, le régime permanent ne pose pas de problèmes à condition de prendre certaines précautions élémentaires; mais des régimes transitoires peuvent se développer en présence de perturbations sur les lignes : il conviendrait de réduire les pertes de un à deux ordres de grandeur pour que le système puisse absorber le contre-coup lié à l'apparition d'un tel régime transitoire.

Les pertes en régime variable, pour un conducteur donné, possédant un état de surface donné, à capacité de transport fixe, varient comme le rapport $\varnothing / J \mathrm{c}$ où $\varnothing$ est le diamètre d'un filament à condition que les filaments soient transposés. Toute la difficulté pour le fabricant de matériau revient donc à produire des matériaux avec des filaments de diamètre le plus faible possible, tout en conservant une valeur élevée de $J \mathrm{c}$ : il y a une opposition évidente entre les deux termes de cette proposition, puisque l'existence d'un courant critique élevé est liée à la présence de défauts structuraux qui limitent la ductilité. Actuellement des conducteurs comportant des filaments de diamètre 10 à $20 \mu$ sont couramment réalisés et les fabricants pensent pouvoir réaliser dans un avenir relativement proche des conduc- teurs de grande longueur comportant des filaments de diamètre 1 à $2 \mu$, résultat déjà acquis sur des échantillons courts. Aller plus loin sera très difficile, et on atteindrait d'ailleurs assez rapidement des tailles de filaments à partir desquelles la matrice introduirait un effet de proximité tout à fait défavorable.

A condition de conserver une densité de courant élevée et un état de surface que les transformations successives ne déprécient pas trop, on peut donc estimer réduire les pertes par un ordre de grandeur, mais ceci semble une limite que les matériaux ne permettront guère de dépasser. Ceci ne signifie pas nécessairement que les applications en régime alternatif soient fermées aux matériaux $\mathrm{NbTi}$ du type filamentaire à matrice conductrice : une optimisation des différents paramètres de composition, de fabrication et de conditionnement de ces matériaux et une conception repensée des machines devraient permettre d'aller au-delà des possibilités évaluées à partir des propriétés mesurées ou extrapolées. Ceci exigerait une compilation de résultats expérimentaux et une coopération très active entre fabricants de matériaux et utilisateurs.

La réalisation sous forme filamentaire des matériaux de type $A_{15}$, et de $\mathrm{Nb}_{3} \mathrm{Sn}$ en particulier, a aussi été tentée [1]. Il faudrait une diminution très sensible du prix de revient de ces matériaux pour qu'on puisse envisager leur utilisation à la place des filamentaires $\mathrm{NbTi}$. Parmi les composés $\mathrm{A}_{15}, \mathrm{Nb}_{3} \mathrm{AlGe}$ ouvre des perspectives intéressantes : en effet son champ critique dépasse $200 \mathrm{kOe}$ dès $14^{\circ} \mathrm{K}$, ce qui laisse espérer des courants critiques élevés à des températures de 12 à $14 \mathrm{o}$. Une utilisation à ces températures correspondrait à une importante économie. Cependant, les matériaux $\mathrm{Nb}_{3} \mathrm{AlGe}$ réalisés sous un conditionnement non filamentaire mais néanmoins compatible avec la construction d'aimants ont manifesté jusqu'ici des performances assez inférieures à celles que l'on pouvait attendre [2].

2.2 LES MATÉRIAUX NOUVEAUX. - Il faut mentionner une initiative pour fabriquer des matériaux supraconducteurs par une technique originale. Il s'agit des imprégnations de fibres de verres poreux par des alliages $\mathrm{PbBiSb}$ réalisées par Watson [3]. Typiquement, les fibres de verre ont des diamètres de quelques dizaines de $\mu$ avec des pores dont la taille varie de 30 à $100 \AA$. Dans de tels matériaux, des champs critiques $H_{c_{2}}(0)$ compris entre 100 et $200 \mathrm{kOe}$ ont pu être déterminés, les densités de courant critique à $4,2^{\circ} \mathrm{K}$ atteignant $10^{4}$ à $10^{5} \mathrm{~A} . \mathrm{cm}^{-2}$. Ces matériaux sont encore imparfaits : la phase métallique est très inhomogène, ce qui explique que les plus faibles densités de courant critiques aient été mesurées dans les fibres de plus faible diamètre; ils ont tendance à s'autodétruire au refroidissement sous l'effet des contraintes associées aux contractions inhomogènes et à chaud par extrusion de la phase métallique. En dépit de ces imperfections liées à leur nouveauté, ils offrent la 
possiblité d'une très grande dispersion de la phase supraconductrice, sans que l'on soit gêné par des effets de proximité dus à la matrice. Leur comportement en régime alternatif est encore inconnu mais pourrait réserver d'agréables surprises.

Ces premiers résultats joints à des perspectives de prix de revient relativement faibles devraient encourager des efforts sur ces matériaux ou d'autres d'un type analogue.

2.3 LES MATÉRIAUX DE COMMUTATION. - Les matériaux de commutation forment une classe relativement à part. Il est admis généralement que pour les applications électrotechniques du type redresseur, ces matériaux doivent être caractérisés par un facteur de mérite $\rho J \mathrm{c}^{2}$ supérieur à $10^{15} \mathrm{~W} / \mathrm{m}^{3}$, où $\rho$ est la résistivité à l'état normal. Alors l'utilisation de la solution supraconductrice serait très compétitive, même dans un contexte non intégré.

Les plus performants des matériaux de cette classe sont les matériaux granulaires (A1) [4] et surtout les $\mathrm{NbN}$ [5], pour lesquels des valeurs de $\rho J \mathrm{c}^{2}$ supérieures à $10^{14} \mathrm{~W} / \mathrm{m}^{3}$ ont été mesurées, proches des valeurs compatibles avec une application compétitive et loin donc d'être dirimantes. Reste, bien sûr, à mettre en œuvre des techniques conduisant à des objets exploitables, avec un conditionnement en couche mince adapté à la réalisation de dispositifs de faible inertie thermique.

2.4 ORIENTATION DES RECHERCHES SUR LES MATÉRIAUX SUPRACONDUCTEURS. - Les limitations des matériaux actuellement disponibles viennent d'être exposées. Pour aller plus loin, pour les applications aux machines tournantes en régime alternatif, pour les dispositifs de commutation, pour d'une manière générale abaisser le seuil de compétitivité technique et économique de la solution supraconductrice, il est nécessaire de mettre au point des matériaux plus performants et moins chers.

Ceci implique que l'effort porte d'abord sur les matériaux déjà étudiés ou même industrialisés. Cet effort, appliqué pour l'essentiel, doit viser le développement de matériaux pour lesquels des propriétés intéressantes ont été mesurées au laboratoire : composites $\mathrm{NbTi}$ à filaments de quelques $\mu$, composites filamentaires $\mathrm{Nb}_{3} \mathrm{Sn}$ et autres $\mathrm{A}_{15}$, rubans $\mathrm{Nb}_{3} \mathrm{AlGe}$ et $\mathrm{NbN}$, etc...

La théorie encore qu'imparfaite, indique dans quelles directions orienter les recherches pour aboutir à des matériaux peut-êtıe plus performants. Quelles sont, en effet, les perspectives d'augmentation de la température ou de la densité de courant critiques ?

Il y a des espoirs modestes, cependant non négligeables, de parvenir à des Tc plus élevées dans des matériaux à couplage électron-phonon. La théorie de Mc Millan [6] fournit la base de ces espoirs : des expériences récentes assez nombreuses ont permis d'en vérifier les fondements, encore que la multiplicité des paramètres entrant en jeu en ait souvent rendu l'inter- prétation incertaine. Cette théorie permet en particulier de prévoir une température critique maximale pour une classe de matériaux donnée : la température critique pourra être relevée pour un corps donné si, toutes choses égales par ailleurs, on sait renforcer sa constante de couplage, ce qui peut être obtenu en le préparant sous une forme instable dans les conditions ordinaires. L'effet est particulièrement important dans les matériaux à faible constante de couplage : par exemple, des couches minces granulaires de $\mathrm{Al}$, élaborées sur support refroidi à basse température ont ainsi exhibé des températures critiques trois à quatre fois supérieures à leurs valeurs normales [4], [7]. Reste à obtenir un effet semblable dans des corps à température de Debye élevée, plus favorables évidemment [8], sous réserve que des modifications de structure électronique associées à la présentation nouvelle ne changent pas dans le mauvais sens les valeurs de la constante de couplage électron-phonon et de la constante de Coulomb.

Il est à noter que les matériaux à grains fins, notamment les couches minces élaborées sur support refroidi, présentent souvent une température critique supérieure à celle du matériau massif. Plusieurs mécanismes peuvent expliquer ce renforcement du couplage [8], sans que l'on ait pu dans tous les cas déterminer le rôle effectif de chacun. Le point principal reste que ce type de matériau est favorable à une élévation de la température critique.

Les recherches de matériaux dans lesquels un couplage électron-électron (intéractions $s$-d, polarisation par des chaînes organiques) peut conduire au phénomène de supraconductivité ou à un renforcement de ce phénomène n'ont été ni inactives, ni stériles. On peut citer par exemple [9] les composés feuilletés $\mathrm{TaS}_{2}$ - aniline et $\mathrm{TaS}_{2}$ pyridine où les couches successives de composants ont des épaisseurs de $6 \AA$ pour $\mathrm{TaS}_{2}$ et 3 à $30 \AA$ pour la matière organique, et qui ont exhibé des températures critiques allant jusqu'à $3,7 \circ \mathrm{K}$ alors que la température critique de $\mathrm{TaS}_{2}$ est de $0,8{ }^{\circ} \mathrm{K}$. Il y a certainement là un encouragement à l'intérêt porté aux systèmes supraconducteurs à une et deux dimensions.

L'augmentation de la densité de courant critique redevient une préoccupation importante, maintenant que la stabilisation des conducteurs a fait des progrès tels que ces conducteurs sont stables sous des courants supérieurs au courant critique. Quelles sont les perspectives dans ce domaine ? La théorie n'apporte que des indications qualitatives car l'efficacité des divers centres d'ancrage comme les mécanismes contrôlant le courant critique sont encore assez mal connus. La recette reste une distribution d'inhomogénéités dont la taille et l'espacement doivent être de l'ordre de la longueur de cohérence. Mais une amélioration sensible des courants critiques passe par une recherche à caractère fondamental. On peut cependant remarquer que les matériaux à grains fins sont de nature à être le siège de densités de courant critique élevées. 
Pour conclure, il semble qu'en dehors des systèmes à une et deux dimensions des progrès peuvent être attendus d'un conditionnement nouveau de matériaux connus. La théorie et les résultats expérimentaux indiquent que les espoirs les plus sérieux d'augmentation de la température, du champ ou de la densité de courant critiques et de réduction des pertes en régime variable sont liés au développement de matériaux inhomogènes composites caractérisés par une division des phases supraconductrices, normales ou isolantes qui soit inférieure à la centaine d’Å. Ces matériaux granulaires ou filamentaires, peuvent être réalisés par les techniques déjà connues, les procédés de couches minces notamment, ou par des méthodes encore peu développées comme celle des verres imprégnés décrite plus haut, ou la technique des eutectiques orientés à laquelle un exposé sera consacré dans le cadre de ce colloque, ou d'autres encore. Ces procédés, souvent appliqués jusqu'ici à des matériaux non supraconducteurs, n'en sont qu'au stade du laboratoire : les supraconducteurs tireront parti de tout progrès général dont ils pourraient bénéficier. Dans le même ordre d'idées, l'étude et le développement des matériaux supraconducteurs à structure fine se trouve actuellement gênés, pour ne pas dire bloqués, par l'absence de techniques d'analyse et de caractérisation localisées à une échelle inférieure à $100 \AA$. Là encore, les progrès attendus, notamment en microscopie électronique, ne pourront qu'accélérer le développement de nouveaux matériaux supraconducteurs. Il y a là une sérieuse motivation à un effort soutenu sur ces techniques.

3. Conclusion. - Selon toute vraisemblance, les applications de la supraconductivité vont connaître dans les prochaines années, un véritable développement industriel. Cette perspective doit favoriser les efforts d'amélioration des matériaux déjà traditionnels et d'innovation vers des matériaux nouveaux dans les directions où la théorie laisse entrevoir des progrès substantiels. Ces progrès passent par la mise au point de techniques de fabrication et de caractérisation de composites à structure fine.

\section{Bibliographie}

[1] Kaufman (A. R.) and Pickett (J. J.), J. App. Phys., $1971,42,58$.

[2] OCtor (H.), Rocher (Y. A.) et Renard (J. C.), Comptes Rendus Congrès International sur les Couches Minces, Cannes, 1970, 399.

Tanaka (E.), Fukuda (T.), Kuma (S.), Yamashita (T.) and ONODERA (Y.), App. Phys. Letters, 1969, 14, 389.

[3] Watson (J. H. P.), J. App. Phys., 1971, 42, 46 ; App. Phys. Letters, 1970, 16, 428.

[4] Cohen (R. W.) and Abelfs (B.), Phys. Rev., 1968, 168, 444.
[5] Gavaler (J. R.), Janocko (M. A.), Patterson (A.) and Jones (C. K.), J. App. Phys., 1971, 42, 54.

Deis (B. W.), Gavaler (J. R.), Hulm (J. K.) and Jones (C. K.), J. App. Phys., 1969, 40, 2163.

[6] Mc Millan (W. L.), Phys. Rev., 1968, 167, 331.

[7] Klein (J.) et Leger (A.), Phys. Letters, 1968, 28A, 134.

[8] AleXeevski (N. E.), Elektrotechnicky Casopis, 1970, 21, 323.

[9] Gamble (F. R.), Di Salvo (F. J.), Klemm (R. A.) and Geballe (T. H.), Science, 1970, 168, 568. 